- Article type: ACME 2015 Themed Paper

- Date written: $4^{\text {th }}$ October 2015

- Number of words: 3518 , Number of figures: 6

\title{
Air losses in compressed air tunnelling; a prediction model
}

\section{Author 1}

- Alireza Ahangar Asr, PhD

- School of Computing, Science and Engineering, University of Salford, Greater Manchester, UK

Author 2

- Akbar Javadi, PhD

- College of Engineering, Mathematics and Physical Sciences, University of Exeter, Devon, UK

Corresponding author:

Alireza Ahangar Asr

E-mail: a.ahangarasr@salford.ac.uk 


\begin{abstract}
Data (measurements) from a tunnel in Munich, Germany was used to develop and validate a model to predict air loss volumes in the process of compressed air tunnelling. In the implemented case study, compressed air was used as a measure to control the groundwater followed by placing a shotcrete lining as temporary support. Evolutionary polynomial regression was used for modelling. EPR is a data-driven method based on evolutionary computing aiming to search for an explicit and structured polynomial model representing air losses. Comparisons made between the actual measurements and the model predictions represented robustness of the suggested model in learning and predicting the behaviour of the system. A sensitivity analysis was also performed on the developed model which revealed the reliability of the model by correctly presenting the expected effect from air permeability of the soil despites in non-homogeneous and layered nature of the geo-materials involved in this case study.
\end{abstract}

\title{
Keywords
}

air loss, tunnelling, evolutionary modelling.

\section{List of notation}

$\mathrm{F} \quad$ is a function in an $\mathrm{m}$-dimensional space and $\mathrm{m}$ is the number of inputs

$X \quad$ is set of inputs

$\theta \quad$ is set of parameters

$y \quad$ is the estimated vector of output of the process

$a_{j} \quad$ is a constant

$f \quad$ is a function defined by the user

$\mathrm{Y}_{\mathrm{N} \times 1}(\theta, Z)$ is the least squares estimate vector of the $N$ target values

$\mathrm{N} \quad$ is number of target values

$\theta_{d \times 1} \quad$ is the vector of $d=m+1$ parameters $a_{j}$ and $a_{0}$

$\theta^{\top} \quad$ is the transposed vector

$Z_{\mathrm{N} \times d} \quad$ is a matrix formed by

I is (unitary vector)

ya is the target experimental value

$y_{p} \quad$ is the model prediction value

A is the air pressure inside the tunnel

B is the product of the average horizontal permeability value multiplied by the face area

C is the product of the average vertical permeability multiplied by the perimeter area

Qtotal is total amount of air lost from a tunnel

Qface is air loss from the tunnel face

Qwall is air loss from tunnel perimeter

Qother is other air losses (ventilation etc.) 


\section{Introduction}

High ground pressures and also complicated and unpredictable soil and rock mix formations makes tunnelling a very challenging process. Tunnelling experts also have the responsibility to minimize the impact of the construction process on the surface environment and particularly settlement problems in densely populated cities which require expensive and time demanding protective measures to be taken for ground improvement that could lead to the project proving financially infeasible. Compressed air has been used as one of the tunnelling techniques that could help decrease some of these problems. In this approach the water in the ground is excluded using compressed air to help construction of the tunnel (Iftimie et al, 2006; El-Nahhas et al, 1991; Dabill et al, 1996).

Applying compressed air as a way to assist tunnelling in under water table coarse soils has gained crucial importance with the construction of major transportation projects, particularly mass transit infrastructure projects and also water supply and sewage disposal systems in many European countries (Kammerer and Semprich, 1999). The open face tunnelling technique immediately followed by supporting the tunnel wall with a layer of sprayed concrete has proved, in many cases, to be an economical and effective measure to exclude water and minimize the ground settlement (Kammerer and Semprich, 1999).

Under ideal circumstances compressed air acts to enhance the stability of a tunnel in three ways: by balancing the external head of water and resisting its entry into the tunnel, by providing a direct reaction to the field forces attempting to displace the soil particles into the tunnel and by drying the soil at the tunnel face and so increasing its effective strength. However, due to the pressure imbalance between the tunnel invert and tunnel crown levels (because of the linear variation of the water pressure and uniform air pressure) the compressed air drives the groundwater back, flows through the ground to the surface and renders a region of the ground around the tunnel face partially saturated. 
The method of compressed air tunneling has been utilised for over a 100 years and has been successfully applied to a wide variety of geological and constructional circumstances and to many important tunneling projects including in Munich, Vienna, Hong Kong, Singapore, and London. Despite having been used for over a century, adoption of a tunnel pressure and the required volume of air to maintain that pressure, has been based on judgement and empirical formulae. The risks associated with leakage of air from tunnels are not addressed by these methods (Javadi, 2006).

As mentioned there are empirical and semi-empirical methods for calculating tunnel pressure and the quantity of air required (Javadi and Snee, 1997). The accuracy of these predictive methods was discussed by Hoad and Gittoes (Hoad and Gittoes, 1992). They showed that the empirical and semi-empirical methods could be appropriate for a first estimate of air losses, but do not account for real influences on air consumption like layered soil conditions, speed of tunnelling and surface conditions. Javadi (Javadi, 2003) and Javadi and Snee (Javadi, and Snee, 2002) also developed a numerical model for predicting air losses in compressed air tunnelling. They implemented the model to study the effect of air flow in the partially saturated ground on volume change and subsequently the settlements, shear strength of the soil and also stability of the tunnel (Javadi and Snee, 2001). They also suggested a relationship to describe time-dependency of the air permeability of shotcrete lining in compressed air tunnelling. Javadi (Javadi, 2006) also proposed a neural network approach for estimation of air losses from tunnels in compressed air tunnelling.

While neural networks have shown to be very efficient in modelling the behaviour of materials they do have shortcomings. One of the drawbacks of neural network is that the optimum structure of ANN (e.g., number of inputs, hidden layers, and transfer functions) must be identified a priori. This is usually done through a trial and error procedure. The other major shortcoming is the black box nature of ANN model and the fact that the relationship between input and output parameters of the system is described in terms of 
a weight matrix and biases that are not accessible to the user. In fact, the black box nature and lack of interpretability have prevented ANNs from achieving their full potential in engineering applications (Javadi et al, 2012).

The total amount of air lost from a tunnel, $Q_{\text {total, }}$ is the sum of three main sources; (a) Air loss from the tunnel face, $Q_{\text {face, }}$ (b) Air loss from tunnel perimeter walls, $Q_{\text {wall }}$ and (c) other losses (ventilation, etc.), $Q_{\text {other. }}$ The third item can be approximated from the size of the air lock, the pressure and frequency of use. However, it is not significant compared to face losses occurring in open ground (Hoad and Gittoes, 1992) or losses from the tunnel perimeter walls. The main factors influencing the air losses from the tunnel face and perimeter walls are the permeability of soil (which is in turn a function of degree of saturation of the soil) and lining (e.g. shotcrete), size and depth of tunnel and air pressure inside the tunnel (Javadi et al., 1999).

In this paper EPR approach is implemented to develop a model to predict air losses from the tunnel in the compressed air tunnelling technique during the construction process. The proposed modelling methodology has previously been successfully implemented into modelling complicated behaviour of engineering materials and systems (AhangarAsr et al, 2014 \& 2015; Javadi et al, 2015).

\section{Evolutionary Polynomial Regression}

Evolutionary polynomial regression (EPR) is a data mining technique that integrates numerical and symbolic regression. The strategy uses polynomial structures to take advantage of their favourable mathematical properties. The key idea behind the EPR is to use evolutionary search for exponents of polynomial expressions by means of a genetic algorithm (GA) engine. This allows (i) easy computational implementation of the algorithm, (ii) efficient search for an explicit expression, and (iii) improved control of the complexity of the expression generated (Giustolisi \& Savic, 2006). EPR is a data-driven method based on evolutionary computing, aimed to search for polynomial structures representing a system. A physical system, having an output y, dependent on a set of inputs $\mathrm{X}$ and parameters $\theta$, can be mathematically formulated as: 
$y=F(\mathbf{X}, \boldsymbol{\theta})$

1.

where $\mathrm{F}$ is a function in an $\mathrm{m}$-dimensional space and $\mathrm{m}$ is the number of inputs. To avoid the problem of mathematical expressions growing rapidly in length with time, in EPR the evolutionary procedure is conducted in the way that it searches for the exponents of a polynomial function with a fixed maximum number of terms. During one execution it returns a number of expressions with increasing numbers of terms up to a limit (the models developed could have equal or smaller number of terms) set by the user to allow the optimum number of terms to be selected. The general form of expression used in EPR can be presented as (Giustolisi \& Savic, 2006):

$y=\sum_{j=1}^{m} F\left(\mathbf{X}, f(\mathbf{X}), a_{j}\right)+a_{0}$

2.

where $y$ is the estimated vector of output of the process; $a_{j}$ is a constant; $F$ is a function constructed by the process; $X$ is the matrix of input variables; $f$ is a function defined by the user; and $\mathrm{m}$ is the number of terms of the target expression. The first step in identification of the model structure is to transfer equation 2 into the following vector form:

$$
Y_{N \times 1}(\theta, Z)=\left[\begin{array}{ll}
I_{N \times 1} & Z_{N \times m}^{j}
\end{array}\right] \times\left[\begin{array}{llll}
a_{0} & a_{1} & \ldots & a_{m}
\end{array}\right]^{T}=Z_{N \times d} \times \theta_{d \times 1}^{T}
$$

3.

where $\mathbf{Y}_{\mathbf{N} \mathbf{1}}[\boldsymbol{\theta}, \mathbf{Z}]$ is the least squares estimate vector of the $N$ target values; $\boldsymbol{\theta}_{\mathrm{d} \times 1}$ is the vector of $\mathrm{d}=\mathrm{m}+1$ parameters $\mathrm{a}_{j}$ and $\mathrm{a}_{0}\left(\boldsymbol{\theta}^{\mathrm{T}}\right.$ is the transposed vector); and $\mathbf{Z}_{\mathbf{N \times d}}$ is a matrix formed by $\mathbf{I}$ (unitary vector) for bias $a_{0}$, and $m$ vectors of variables $Z_{j}$. For a fixed $j$, the variables $Z_{j}$ are a product of the independent predictor vectors of inputs, $\mathbf{X}=\left[\begin{array}{llll}\mathbf{X}_{1} & \mathbf{X}_{2} & \ldots & \mathbf{X}_{\mathbf{k}}\end{array}\right]$.

In general, EPR is a two-stage technique for constructing symbolic models. Initially, using standard genetic algorithm (GA), it searches for the best form of the function structure, i.e. a combination of vectors of independent inputs, $\mathbf{X}_{\mathbf{s}}=[1, \ldots, \mathbf{k}]$, and secondly it performs a least squares regression to find the adjustable parameters, $\theta$, for each combination of inputs. In this way a global search algorithm is implemented for both the best set of input combinations and related exponents simultaneously, according to the user-defined cost function (Giustolisi \& Savic, 2006). The adjustable parameters, $a_{j}$, are evaluated by means of the linear least squares (LS) 
method based on minimization of the sum of squared errors (SSE) as the cost function. The SSE function, which is used to guide the search process towards the best fit model, is:

$\mathbf{S S E}=\frac{\sum_{i=1}^{N}\left(y_{a}-y_{p}\right)^{2}}{N}$

4.

where $y_{a}$ and $y_{p}$ are the target experimental and the model prediction values respectively. The global search for the best form of the EPR equation is performed by means of a standard GA over the values in the user defined vector of exponents. The GA operates based on Darwinian evolution which begins with random creation of an initial population of solutions (Zeng et al, 2015; Johari et al, 2011; Javadi et al, 2005). Each parameter set in the population represents chromosomes of the individuals. Each individual is assigned a fitness based on how well it performs in its environment. Through crossover and mutation operations, with the probabilities Pc and $\mathrm{Pm}$ respectively, the next generation is created. Fit individuals are selected for mating, whereas weak individuals die off. The mated parents create a child (offspring) with a chromosome set which is a mix of parents' chromosomes. In EPR integer GA coding with single point crossover is used to determine the location of the candidate exponents (Giustolisi \& Savic, 2006).

The EPR process stops when the termination criterion, which can be either the maximum number of generations, the maximum number of terms in the target mathematical expression or a particular allowable error, is satisfied. A typical flow diagram for the EPR procedure is illustrated in Figure 1.

\section{Contributing parameters and modelling procedure}

The Feldmoching tunnel, U8 N-8 in Munich, Germany, was used as a case study. The construction of the $635 \mathrm{~m}$ long Feldmoching tunnel, U8 N-8, was started in July 92 and finished in February 94. The tunnel was constructed using a top heading and bench method. Compressed air was used to control the groundwater and shotcrete was used as temporary support. The final concrete lining was installed in free air after completion of the tunnel. Detailed information about the tunnel construction can be found in (Javadi, 2006). 
Parameters used as input parameters in the model development process included, perimeter area of the tunnel, average horizontal permeability of the soil layers, average vertical permeability of the soil layers, the air pressure required and face area of the tunnel. The output parameter was considered to be the air loss from the face and perimeter areas of the tunnel $\left(Q_{t o t a l}\right)$.

The data set comprised 36 lines of data. Data was divided into training and testing sets. The training data representing approximately $90 \%$ of the whole set was used to develop the EPR model. The testing (verification) data set (the remaining $10 \%$ of the data lines) was however kept unseen to EPR during the model development process and has been used to examine the generalization capabilities of the developed model in giving accurate predictions for the cases not previously experienced by EPR.

Various combinations of training and testing data sets were initially developed. It was checked to make sure that all parameter values in the testing data sets were always within the range of data chosen to be used for training and developing the EPR models to avoid extrapolation. A statistical analysis was performed on the data to select the most statistically consistent training and testing sets combination to be utilized in the development of the presented model. The aim of the analysis was to ensure that the statistical properties of the data in each of the subsets were as close to the others as possible and thus represented the same statistical population. The mean and standard deviation values were calculated for every single contributing parameter and for the training and testing datasets for each combination and the one for which these statistical values were the closest in the training and testing data sets was chosen to be used in training and testing stages in the EPR model development process.

By completing the training stage in the development process the following equation was developed by EPR to represent the air losses through the tunnel. The model was chosen based of the performance considering the verification phase and also the parametric study (detailed below):

$Q_{\text {total }}=513.33 A^{2}+1.37 \times 10^{12} B^{2} \cdot C^{2}-840.14$

5.

In the presented EPR model (Equation 5) $\mathrm{A}$ is the air pressure applied; $\mathrm{B}$ is defined as the product of the average horizontal permeability value multiplied by the face area of the tunnel and $C$ is the product of the average vertical permeability multiplied by the perimeter area of the tunnel. Figure 
2 shows the Geology of the tunnel route. Figures 3 and 4 show a comparison between the prediction results of the EPR model for training and validation data and the actual measurements respectively.

\section{Parametric study}

A sensitivity analysis was conducted on the developed EPR model to investigate into the trend and level of impact from each contributing parameter on predicted air losses through the tunnel in construction. All the input parameters but the one being examined were kept constant and the model predictions for different values - within the maximum and minimum values of the parameter in the database within the available range of data - of the parameter under study were investigated. Figures 5 and 6 represent the combined sensitivity analysis results conducted on the effects of horizontal and vertical permeability as well as face and perimeter areas of the tunnel on the volume of air losses.

\section{Conclusions}

An evolutionary-based EPR model for predicting air losses in compressed air tunnelling was developed and validated using a field measurement database from literature. The data used to validate the developed model was not used during the model production process to ensure unbiased judgment on the performance and generalization capabilities of the model in making accurate predictions of the air loss phenomenon. The model prediction results were compared to the actual measured data. Comparison of the results showed that the model had been able to capture the data patterns and underlying relations between input and output parameters and provide accurate predictions for total air losses through the tunnel during the construction process. A parametric study was conducted to evaluate the combined effects of the air permeability in both horizontal and vertical directions with the perimeter and face areas on air loss volumes. The results revealed that, as expected, increasing air permeability in both horizontal and vertical directions combined with the associated pressure contact areas will lead to increase in total air loss volumes. The evolutionary polynomial regression provides multiple models to reflect the behaviour of systems. This enables the user to apply the existing knowledge and understanding 
of the behaviour to choose the best possible models on the basis of their complexity and performance in predicting the expected behaviours. Additional data acquired as the tunnelling process progresses can also be used to retrain and redevelop the EPR model on real-time basis to enhance accuracy and reliability of the predictions and ensure time efficiency and financial feasibility.

\section{References}

Ahangar-Asr A, Javadi A A, Johari, A and Chen Y (2014) Lateral load bearing capacity modelling of piles in cohesive soils in undrained conditions; an intelligent evolutionary approach. Applied Soft Computing; an international journal 24: 822-828.

Ahangar-Asr A, Javadi A A and Khalili N (2015) A new approach to thermo-mechanical modelling of the behaviour of unsaturated soils. International Journal for Numerical and Analytical Methods in Geomechanics 39: 539-557.

Dabill D W, Groves J A and Lamont D R (1996). The Effect of Pressure on Portable Gas Monitoring Equipment during Compressed Air Tunnelling. Ann. Occup. Hyg. 40(1):11-28.

El-Nahhas F, El-Kadi F and Shalaby A (1991). Field measurements during construction of a compressed air tunnel in Cairo. Tunnelling and Underground Space Technology 6(1): 123127.

Giustolisi O and Savic D A (2006). A Symbolic Data-driven Technique Based on Evolutionary Polynomial Regression. Journal of Hydroinformatics 8(3): 207-22.

Hoad R S and Gittoes G P (1992). Estimation of compressed air losses. In: Proceedings of the Conference on Engineering and Health in Compressed Air Work CIRIA157-17.

Iftimie T, Suhane S, Carstea D, Takaks P A and Dilaveris G (2006). Tunneling under compressed air through immersed soft soil strata. Tunnelling and Underground Space Technology 21: 356357.

Javadi A A (2006). Estimation of air losses in compressed air tunnelling using neural network. Tunneling and Underground Space Technology 21: 9-20.

Javadi A A (2003). Numerical and experimental study of compressed air tunnelling- A Chapter in: Numerical Analysis and Modelling in Geomechanics (Bull JW (Ed)). SPON Press.

Javadi A A, Ahangar-Asr A, Johari A, Faramarzi A and Toll D G (2012). Modelling Stress-Strain and Volume Change Behaviour of Unsaturated Soils using an Evolutionary Based Data Mining Technique, an Incremental Approach. Engineering Applications of Artificial Intelligence Journal 25(5): 926-933.

Javadi A A, Farmani R and Tan T P (2005). A hybrid intelligent genetic algorithm. Advanced Engineering Informatics 19: 255-262.

Javadi A A, Farmani R, Toropov V V and Snee CPM (1999) Identification of parameters for air permeability of shotcrete tunnel lining using a genetic algorithm. Journal of Computers and Geotechnics 25: 1-24. 
Javadi A A, Hussain M, Ahangar-Asr A, Farmani, R (2015) A surrogate model for simulationoptimization of aquifer systems subjected to seawater intrusion. Journal of Hydrology 532 : 542-554.

Javadi A A and Snee C P M (1997) New method in predicting air losses in compressed air tunnelling. In: Proceedings of the Tunnelling '97 Conference, Published by the Institution of Mining and Metallurgy London 2-4 September 57-69.

Javadi A A and Snee C P M (2002). Numerical modelling of compressed air tunnelling. International Journal of Geomechanics 2 (4), 399-417.

Javadi A A and Snee C P M (2001). The effect of air pressure on the shear strength of soil as a consequence of compressed air tunnelling. Canadian Geotechnical Journal 38 (6), 11871200.

Johari A, Javadi A A and Habibagahi G (2011). Modelling the mechanical behaviour of unsaturated soils using a genetic algorithm-based neural network. Computers and Geotechnics 38: 2-13.

Kammerer $G$ and Semprich S (1999). Tunnelling under compressed air - the experience on site and a laboratory test. In: Proceedings of the 12th European Conference on Soil Mechanics and Geotechnical Engineering, 6-10 June, Amsterdam.

Zeng P, Jimenez R and Jurado-Pina R (2015). System reliability analysis of layered soil slopes using fully specified slip surfaces and genetic algorithms. Engineering Geology 193 : 106-117.

\section{Figure captions}

Figure 1: Evolutionary polynomial regression modelling flowchart

Figure 2: Geological setting of the tunnel route

Figure 3: EPR predictions against field measurement values of total air loss for EPR training data Figure 4: EPR predictions against field measurement values of total air loss for EPR testing data Figure 5: Parametric study results - combined effect of horizontal air permeability and face area Figure 6: Parametric study results - combined effect of vertical air permeability and perimeter area 